\title{
Tunable near-infrared and visible-light transmittance in nanocrystal-in-glass composites
}

\author{
Anna Llordés ${ }^{1}$, Guillermo Garcia ${ }^{1}$, Jaume Gazquez ${ }^{2}$ \& Delia J. Milliron ${ }^{1}$
}

Amorphous metal oxides are useful in optical ${ }^{1,2}$, electronic ${ }^{3-5}$ and electrochemical devices ${ }^{6,7}$. The bonding arrangement within these glasses largely determines their properties, yet it remains a challenge to manipulate their structures in a controlled manner. Recently, we developed synthetic protocols for incorporating nanocrystals that are covalently bonded into amorphous materials ${ }^{8,9}$. This 'nanocrystalin-glass' approach not only combines two functional components in one material, but also the covalent link enables us to manipulate the glass structure to change its properties. Here we illustrate the power of this approach by introducing tin-doped indium oxide nanocrystals into niobium oxide glass $\left(\mathrm{NbO}_{\mathrm{x}}\right)$, and realize a new amorphous structure as a consequence of linking it to the nanocrystals. The resulting material demonstrates a previously unrealized optical switching behaviour that will enable the dynamic control of solar radiation transmittance through windows. These transparent films can block near-infrared and visible light selectively and independently by varying the applied electrochemical voltage over a range of 2.5 volts. We also show that the reconstructed $\mathrm{NbO}_{x}$ glass has superior properties-its optical contrast is enhanced fivefold and it has excellent electrochemical stability, with 96 per cent of charge capacity retained after 2,000 cycles.

Enhanced properties have been demonstrated when nanocrystalline precipitates form within a glassy matrix ${ }^{10-15}$. These crystalline domains can harden bulk glasses ${ }^{10}$, introduce optical nonlinearity ${ }^{11}$ or lead to anomalous transparency ${ }^{12}$. Anecdotal evidence also suggests that ion transport may be enhanced at crystal-glass interfaces. For instance, when $\mathrm{LiAlSiO}_{4}$ was partially crystallized, fast relaxation of ions was observed ${ }^{13}$, whereas precipitating $\mathrm{TiO}_{2}$ nanocrystals within mesoporous $\mathrm{P}_{2} \mathrm{O}_{5}$ glass led to high-power lithium $(\mathrm{Li})$ ion battery electrodes ${ }^{14}$. Structural modifications at crystal-glass interfaces have been proposed to explain such observations, but no direct evidence has been reported owing to the limited structural and compositional control offered by those preparation methods. Conventional methods of preparing glass ceramics rely on thermally induced phase separation and in situ crystallization processes, which are very complex to control experimentally ${ }^{16}$. The timetemperature parameter space for selective nanocrystallization is typically narrow and strongly influenced by the glass composition, structure and thermal history ${ }^{16}$. Therefore, determining the heat-treatment conditions required for selective crystallization of a desired composition phase with high control over the interface area becomes not only a challenging and tedious task, but also a nearly impossible task for complex multicomponent stoichiometries (for example, doped nanocrystals). Here we show how to control these characteristics by using well-defined colloidal nanocrystals that are post-synthetically embedded in and chemically linked to glass.

In our colloidal approach, chemical bonds are made by combining ligand-stripped $\mathrm{Sn}$-doped $\operatorname{In}_{2} \mathrm{O}_{3}$ (ITO) nanocrystals ${ }^{17,18}$ and polyniobate clusters (POMs) in solution'. This process results in POM-stabilized nanocrystal dispersions (Fig. 1a, Supplementary Fig. 2), from which films are deposited and then thermally annealed in air, at $T=400{ }^{\circ} \mathrm{C}$ (Fig. 1b).X-ray diffraction (XRD) analysis of as-deposited and annealed

films shows that the crystalline molecular POMs condense to an amorphous $\mathrm{NbO}_{x}$ matrix (Supplementary Fig. 3). In addition, XRD patterns (Fig. 1c, d) and scanning transmission electron microscopy cross-sectional images (Fig. 1e, f) confirm the amorphous nature of the $\mathrm{NbO}_{x}$ matrix and that the embedded ITO nanocrystals remain highly crystalline. This synthetic approach is very flexible, permitting the selection of unusual combinations of components to tailor functionality. For instance, ITO nanocrystals and amorphous $\mathrm{NbO}_{x}$ were specifically selected for their spectrally distinct electrochromic response. The volume fraction was finely tuned over a broad range, from $0 \%$ to $69 \%$, by simply adding additional POM to the aqueous POM-stabilized nanocrystal dispersion (Fig. 1a). This tunability is key to revealing the reconstruction of the $\mathrm{NbO}_{x}$ glass structure induced by its covalent linkage to ITO nanocrystals.

To evaluate the structure of the glass, the Raman spectrum of a pure amorphous $\mathrm{NbO}_{x}$ film was compared to those of ITO-in- $\mathrm{NbO}_{x}$ with different nanocrystal-glass interfacial areas (Fig. 2). Vibrational bands of the distorted $\left[\mathrm{NbO}_{6}\right]$ octahedra that dominate the structure of $\mathrm{NbO}_{x}$
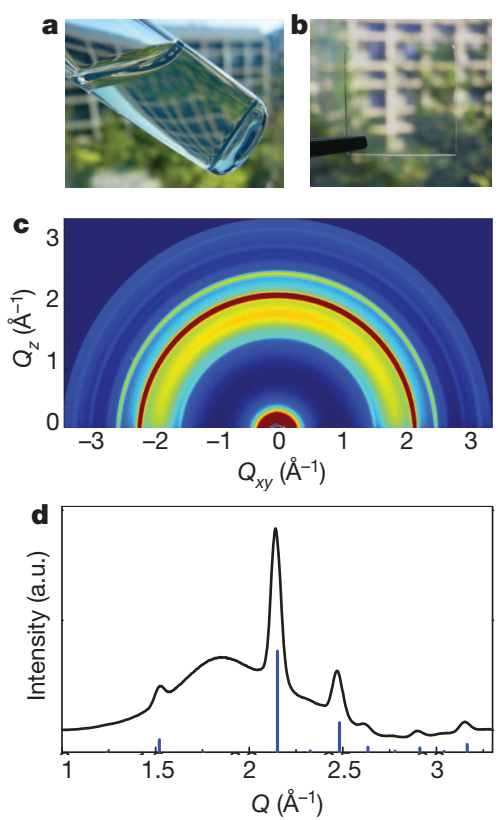

Figure $1 \mid$ Nanocrystal-in-glass film preparation and structural characterization. a, POM-stabilized ITO aqueous dispersion, that is, POM; $\left[\mathrm{N}\left(\mathrm{CH}_{3}\right)_{4}\right]_{6} \mathrm{Nb}_{10} \mathrm{O}_{28}$. b, ITO-in- $\mathrm{NbO}_{x}$ film on a glass substrate. On annealing, the anionic $\left[\mathrm{Nb}_{10} \mathrm{O}_{28}\right]^{6-}$ clusters condense (Supplementary Fig. 3) and the $\left[\mathrm{N}\left(\mathrm{CH}_{3}\right)_{4}\right]^{+}$counterions thermally decompose ${ }^{9}$. c, Two-dimensional XRD image of a ITO-in- $\mathrm{NbO}_{x}$ film. $\mathbf{d}, \mathrm{XRD}$ pattern obtained after integrating along the out-of-plane scattering direction $Q_{z}$. The indexed diffraction lines result from randomly oriented ITO nanocrystals (cubic $\operatorname{In}_{2} \mathrm{O}_{3}$ bixbyite structure) whereas the broad peak at $Q=1.85 \AA^{-1}$ is assigned to amorphous $\mathrm{NbO}_{x}$. e, f, Low- and high-resolution cross-sectional scanning transmission electron microscopy $Z$-contrast images (see Methods). 


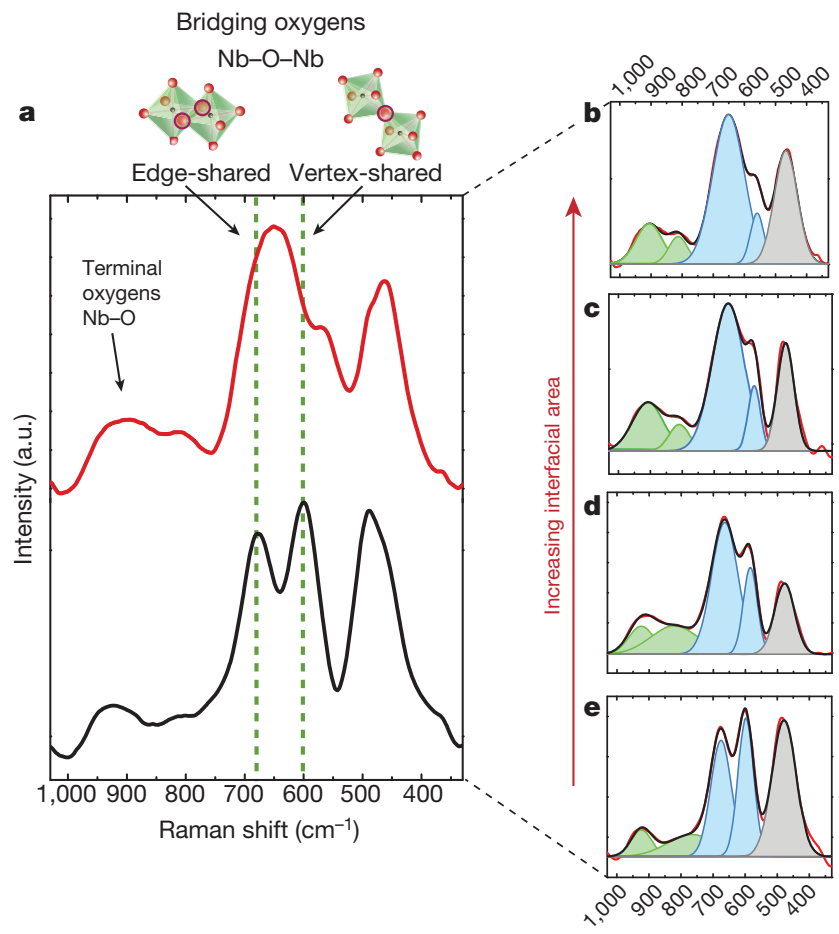

Figure $2 \mid$ Raman analysis probing the reconstruction of a $\mathrm{NbO}_{x}$ glass matrix when linked to nanocrystals. a, Raman spectra of pure $\mathrm{NbO}_{x}$ (black) and ITO-in- $\mathrm{NbO}_{x}$ nanocomposite (red) with 64 vol.\% ITO. b-e, Fitted Raman spectra (experimental data in red, sum of fits in black). From bottom to top are pure $\mathrm{NbO}_{x}$ followed by nanocomposites with increasing ITO volume fraction $(0.48,0.52$ and 0.64$)$. Deconvolved peaks (grey) arise non-specifically from ITO, $\mathrm{NbO}_{x}$ and from the underlying glass substrate. Blue and green peaks specifically arise from $\mathrm{NbO}_{x}$ (bridging oxygens and terminal oxygens, respectively)

are readily observed in the range $500 \mathrm{~cm}^{-1}$ to $1,000 \mathrm{~cm}^{-1}$ and changes in their peak positions and relative intensities track with the interfacial area. This information was used to identify the structural changes in the glass as a result of linking to the nanocrystals.

The topological structure of $\mathrm{NbO}_{x}$ glass is characterized by how the $\left[\mathrm{NbO}_{6}\right]$ octahedral units are connected, namely through vertices and along edges, as well as by the degree of connectivity in the disordered network. Given the higher electrostatic repulsions in edge-sharing topologies compared to vertex-sharing ${ }^{19}$, distinctive vibrational modes for the bridging oxygens can be observed ${ }^{20}$. By deconvolving these bridging oxygen bands $\left(550 \mathrm{~cm}^{-1}\right.$ to $\left.750 \mathrm{~cm}^{-1}\right)$, we find that highly distorted edge-sharing $\left[\mathrm{NbO}_{6}\right]$ are prevalent in the nanocrystal-in-glass films, whereas pure $\mathrm{NbO}_{x}$ contains more regular, vertex-sharing arrangements (Fig. 2, Table 1, Supplementary Fig. 4). In addition, the relative intensity of Raman peaks at higher frequencies $\left(750 \mathrm{~cm}^{-1}\right.$ to $\left.950 \mathrm{~cm}^{-1}\right)$ increases at higher nanocrystal loading, suggesting reduced connectivity of the glassy network. In particular, the highest-frequency peak, $900 \mathrm{~cm}^{-1}$ to $925 \mathrm{~cm}^{-1}$, is assigned to terminal Nb-O bonds. The ratio between these terminal bonds and bridging ones is greater in the nanocomposites (Fig. 2, Table 1, Supplementary Fig. 4), which we attribute to internal interruption of the polymerized network. Generally, the degree of connectivity in oxide glasses can be reduced by addition of network modifiers or glass intermediates ${ }^{21}$. In our case, we suggest that indium (In) and tin (Sn) from the ITO nanocrystals diffuse locally into the $\mathrm{NbO}_{x}$ glass during thermal processing. There they disrupt the glassy network, inducing chain breaking and thus reducing the network connectivity. Indeed, XRD patterns of films processed at increasing temperatures $\left(400{ }^{\circ} \mathrm{C}-550{ }^{\circ} \mathrm{C}\right)$ showed broader ITO peaks (Supplementary Fig. 5b), pointing to progressive interfacial dissolution of ITO in the $\mathrm{NbO}_{x}$ matrix. The presence of a ternary $\mathrm{InNbO}_{4}$ phase following high-temperature annealing $\left(>550^{\circ} \mathrm{C}\right)$ (Supplementary Fig. $5 \mathrm{c}, \mathrm{d}$ ) further supports the idea that In and probably $\mathrm{Sn}$ diffuse into the $\mathrm{NbO}_{x}$ matrix adjacent to the nanocrystals. Hence, the structure of the $\mathrm{NbO}_{x}$ glass is profoundly altered by covalent linking to ITO nanocrystals, becoming highly distorted, less interconnected and interfacially doped (Fig. 3).

The consequence of linking ITO nanocrystals within a $\mathrm{NbO}_{x}$ glass matrix is that a unique electrochromic optical switching functionality can be realized (Fig. 4). As explained below, this new optical behaviour not only reflects the individual properties of the two components, but also is quantifiably superior as a result of the reconstruction of the amorphous material.

Amorphous metal oxides are leading candidates for dynamically controlling solar radiation transmitted through 'smart' windows by means of their electrochromic properties. Electrochromism is a reversible change of optical transmittance in response to electrochemical charging and discharging. After more than 40 years of research in electrochromics ${ }^{22}$, spectral selectivity, that is, independent modulation of visible and near-infrared (NIR) radiation, is still considered a holy grail' for reducing the energy needed to light and thermally regulate building interiors. For instance, others have proposed combining thermochromic and electrochromic materials to modulate NIR and visible light, respectively ${ }^{23}$, although fully integrated devices based on this concept have yet to be reported. We took a step towards this goal through our recent demonstration that charged ITO nanocrystals selectively block NIR light through a plasmonic electrochromic effect ${ }^{24}$ (Fig. 4f). In contrast, amorphous transition metal oxides such as $\mathrm{NbO}_{x}$ modulate mostly visible light under electrochemical bias (Fig. 4a). Now, by linking these two materials and controlling the applied voltage, we find that the transmittance can be modulated in both spectral bands (Fig. 4a-g, Supplementary Fig. 1). The nanocrystal-in-glass coating switches progressively between three optical states: fully transparent (at $4 \mathrm{~V}$ versus Li), selectively NIR blocking (around $2.3 \mathrm{~V}$ ) and broadband blocking of visible and NIR (1.5 V). Thus, solar radiation can now be dynamically modulated with spectral selectivity (Supplementary Fig. 1). The modulation of NIR light derives from the free electrons in the ITO, whose concentration reaches approximately $1.9 \times 10^{21} \mathrm{~cm}^{-3}$ at $2.3 \mathrm{~V}$ (Supplementary Information and Supplementary Fig. 9). Ultimately, the modular construction of our materials will facilitate even greater modulation of NIR transmittance as new plasmonic nanocrystals are developed with a higher free-electron concentration and a broader dynamic optical range ${ }^{25}$.

Remarkably, the modest visible-light modulation of amorphous $\mathrm{NbO}_{x}$ (Fig. 4a) was greatly exceeded by that of the nanocrystal-in-glass composites (Fig. $4 \mathrm{~b}-\mathrm{d}$ ), indicating that the reconstructed $\mathrm{NbO}_{x}$ glass is far more electrochemically active. The optical contrast in the visible range monotonically increased with nanocrystal content up to $\sim 43 \%$ ITO,

Table 1 | Peak-fitting analysis of Raman spectra

\begin{tabular}{|c|c|c|c|c|c|}
\hline \multirow{2}{*}{$\begin{array}{l}\text { ITO nanocrystals } \\
\text { (vol.\%) }\end{array}$} & \multirow{2}{*}{$\begin{array}{l}\text { Specific interface area } \\
\qquad\left(\mathrm{nm}^{-1}\right)\end{array}$} & \multicolumn{2}{|c|}{ Peak position $\left(\mathrm{cm}^{-1}\right)$} & \multicolumn{2}{|c|}{ Peak area ratios } \\
\hline & & $\begin{array}{l}\text { Bridging oxygens in } \\
\text { edge-shared }\left[\mathrm{NbO}_{6}\right]\end{array}$ & $\begin{array}{l}\text { Bridging oxygens in } \\
\text { vertex-shared }\left[\mathrm{NbO}_{6}\right]\end{array}$ & $\begin{array}{l}\text { Edge-shared/ } \\
\text { vertex-shared }\end{array}$ & $\begin{array}{c}\text { Bridging oxygens / terminal } \\
\text { oxygens }\end{array}$ \\
\hline 64 & 0.85 & 651 & 559 & 6.9 & 5.1 \\
\hline 52 & 0.69 & 656 & 574 & 5.9 & 3.7 \\
\hline 48 & 0.64 & 664 & 585 & 2.9 & 6.7 \\
\hline 0 & 0 & 675 & 598 & 1.1 & 8.4 \\
\hline
\end{tabular}




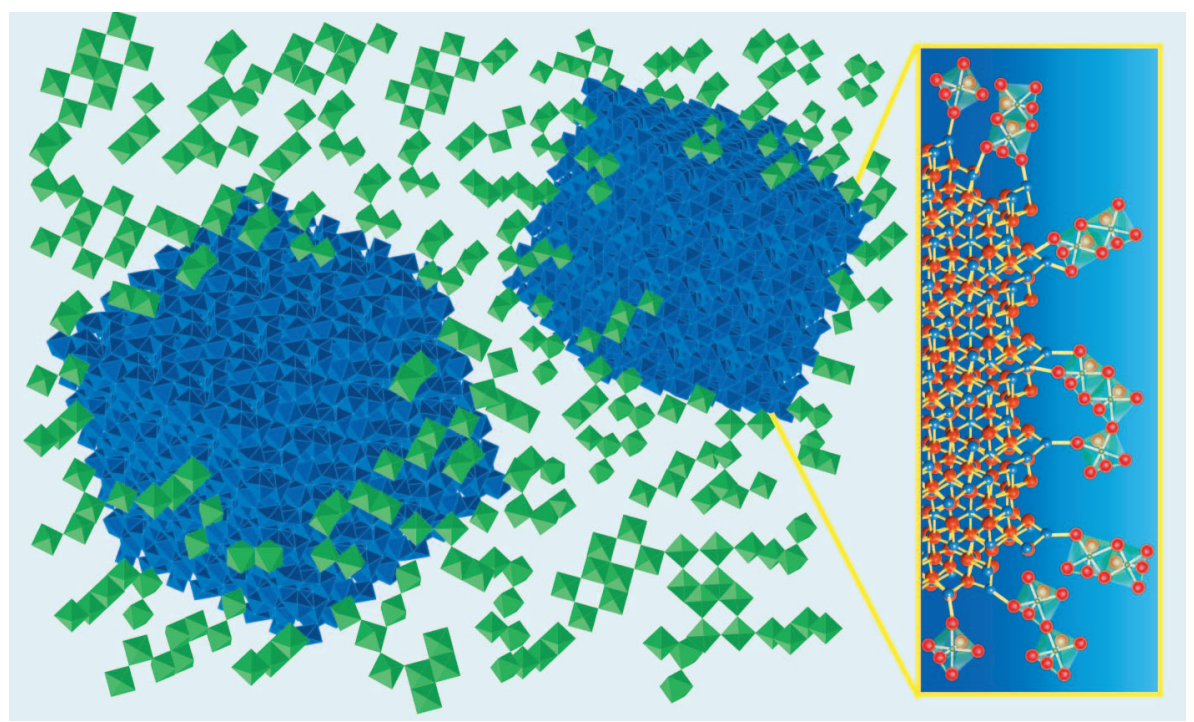

Figure 3 ITO nanocrystals covalently linked to amorphous $\mathrm{NbO}_{x}$. The $\left[\mathrm{NbO}_{6}\right]$ octahedral units are shown in green and the ITO nanocrystals are blue.

reaching a value that is five times greater than that of pure $\mathrm{NbO}_{x}$ (Fig. 4h). At higher nanocrystal loading, the contrast decreased again, indicating that the enhancement is maximized when the average nanocrystal spacing is around $0.7 \mathrm{~nm}$ (Supplementary Information). At this high interface density $\left(0.54 \mathrm{~nm}^{-1}\right)$, the structure of the $\mathrm{NbO}_{x}$ is rearranged throughout, effectively resulting in a new polymorph that exhibits functional characteristics distinct from those of the bulk glass, in fact resembling

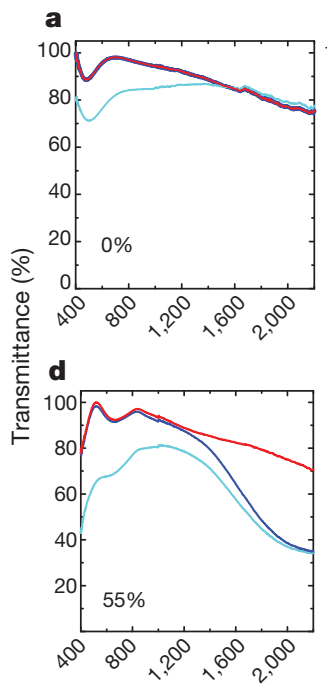

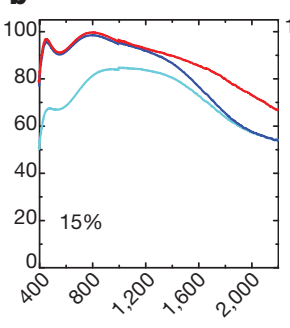

e

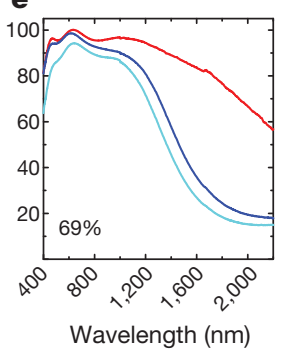

c

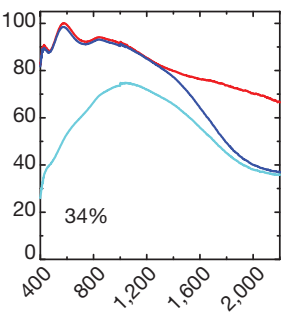

$\mathbf{f}$

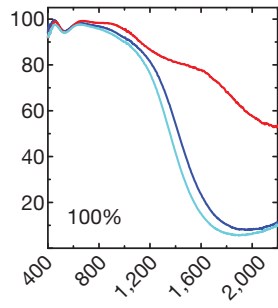

In the inset, the niobium atoms are located at the centre of the green octahedra, oxygen atoms are red and indium atoms are blue.

more the stronger electrochromism seen in the high-temperature pseudohexagonal crystalline phase of $\mathrm{Nb}_{2} \mathrm{O}_{5}$ (known as the TT-phase) (ref. 26).

The reconstructed, nanocrystal-linked amorphous $\mathrm{NbO}_{x}$ network is also highly stable to insertion and extraction of Li ions. The electrochemical cycling stability exceeds that of either individual component; only $4 \%$ charge capacity was lost after 2,000 cycles (between $4 \mathrm{~V}$ and $1.5 \mathrm{~V}$ ), whereas pure $\mathrm{NbO}_{x}$ and especially ITO nanocrystal films degraded substantially (20\% and $85 \%$ capacity losses, respectively) (Supplementary Fig. 7a). In agreement with the electrochemical stability of ITO-in$\mathrm{NbO}_{x}$ composites, the dual-band optical modulation characteristics were maintained after cycling (Supplementary Fig. 7b). We suggest that the enhancements in both optical contrast and stability are related to the more open network structure of the reconstructed glass, which facilitates ion insertion and extraction and helps to relax the stress induced by these processes. Consistent with this hypothesis, the charge capacity increases proportionally with the optical contrast (Supplementary Fig. 8).

Linking undoped $\mathrm{In}_{2} \mathrm{O}_{3}$ nanocrystals to $\mathrm{NbO}_{x}$, instead of Sn-doped $\mathrm{In}_{2} \mathrm{O}_{3}$ nanocrystals, similarly enhanced the optical contrast arising from the matrix (Fig. 4h, Supplementary Fig. 10), which eliminates the possibility that potential electrochemically active Sn species could play a part in such enhancement. Instead, this result further confirms that the optical contrast enhancement is related to a structural reconstruction of the $\mathrm{NbO}_{x}$ matrix as a consequence of the covalent linkage to the embedded nanocrystals. Very probably, similar rearrangements of the glass structure could be involved in the enhanced ionic transport properties previously reported ${ }^{13,14,27}$ in diverse nanocrystal-in-glass materials made by a variety of methods. Therefore, reconstructing glass

Figure $4 \mid$ Tunable dual-band solar control and optical contrast enhancement in nanocrystal-in-glass films. a-g, Transmittance spectra under applied electrochemical voltage $(4 \mathrm{~V}$ in red, $2.3 \mathrm{~V}$ in blue and $1.5 \mathrm{~V}$ in cyan, versus $\mathrm{Li} / \mathrm{Li}^{+}$), of ITO-in- $\mathrm{NbO}_{x}$ with different ITO nanocrystal content. Pure $\mathrm{NbO}_{\mathrm{x}}(\mathbf{a})$ and pure ITO nanocrystal films $(\mathbf{f})$ are shown as reference. The specific interfacial area was tuned from $0 \mathrm{~nm}^{-1}$ to $0.92 \mathrm{~nm}^{-1}$ by varying the ITO nanocrystal percentage by volume between $0 \%$ and $69 \%$ at a constant size of $4.8 \mathrm{~nm} \pm 0.8 \mathrm{~nm}$, chosen to maximize the electrochromic respons $\mathrm{e}^{24}$. The atomic percentage of $\mathrm{Sn}$ in ITO was $12.2 \pm 0.1 \%$ and film thickness was $150 \pm 35 \mathrm{~nm}$. g, Transmittance spectra of a typical ITO-in- $\mathrm{NbO}_{\mathrm{x}}$ film, with intermediate voltages shown in grey. $\mathbf{h}$, Change in absorbance $(\triangle A)$ at $\lambda=500 \mathrm{~nm}$ between $1.5 \mathrm{~V}$ and $4 \mathrm{~V}$, versus volume fraction of ITO nanocrystals $\left(f_{\text {Iто }}\right) . \Delta A$ is normalized by $\mathrm{NbO}_{x}$ volume fraction. The $\Delta A$ of a control sample with undoped $\operatorname{In}_{2} \mathrm{O}_{3}$ nanocrystals is also plotted (star symbol). Photographs of charged films with $f_{\mathrm{ITO}}=0$ and $f_{\mathrm{ITO}}=0.34$ are inset. 
via nanocrystal linking could represent a general strategy for the manipulation of ion transport.

In essence, this nanocrystal-in-glass approach represents the next step in the evolution of the concept of linking building units together to construct new functional materials. Analogously, organic molecules are held together by weak, dynamic bonds to form supramolecular assemblies ${ }^{28}$, metal ions are covalently bonded to organic struts to form metal-organic frameworks ${ }^{29}$ and nanocrystals are linked directly to each other in mesoporous architectures ${ }^{30}$. We have shown that when nanocrystals are covalently bonded to glass, the linking plays a far more active part than in these earlier constructs: the glass structure can be profoundly changed, generating new amorphous structural motifs with distinct functionality.

\section{METHODS SUMMARY}

Nanocrystal-in-glass (ITO-in- $\mathrm{NbO}_{x}$ ) composite films were prepared from aqueous colloidal solutions, containing polyoxometalate clusters bonded to the nanocrystals' surface. On solution deposition (spin coating) and thermal annealing $\left(400{ }^{\circ} \mathrm{C}\right.$ in air), polyoxometalates condense into an amorphous oxide matrix, which remains covalently linked to the embedded nanocrystals. The volume fraction of nanocrystals in the nanocomposite films was tuned by varying the polyoxometalate molar concentration in the initial colloidal solution. XRD and scanning transmission electron microscopy were used for structural characterization of the films at different stages of the thermal process. In addition, films with different interfacial densities were analysed by Raman spectroscopy to systematically follow structural changes in the amorphous matrix induced by the presence of nanocrystals. In parallel, the electrochromic properties were studied by means of spectroelectrochemical measurements. Transmittance spectra were acquired in situ, under applied voltage, by placing the film as working electrode in a liquid spectroelectrochemical cell ( $\mathrm{Li}$ foils as counter/reference electrodes and $\mathrm{LiClO}_{4} /$ propylene carbonate as electrolyte).

Full Methods and any associated references are available in the online version of the paper.

\section{Received 14 December 2012; accepted 12 June 2013.}

1. Lines, M. E. Oxide glasses for fast photonic switching-a comparative study. J. Appl. Phys. 69, 6876-6884 (1991).

2. Kim, S. H. \& Yoko, T. Non-linear optical properties of $\mathrm{TeO}_{2}$-based glasses: $\mathrm{MO}_{(\mathrm{X})}$ $\mathrm{TeO}_{2}(\mathrm{M}=\mathrm{Sc}, \mathrm{Tl}, \mathrm{V}, \mathrm{Nb}, \mathrm{Mo}, \mathrm{Ta}$, and $\mathrm{W}$ ) binary glasses. J. Am. Ceram. Soc. 78, 1061-1065 (1995).

3. Nomura, K. et al. Room-temperature fabrication of transparent flexible thin-film transistors using amorphous oxide semiconductors. Nature 432, 488-492 (2004).

4. Arhammar, C. et al. Unveiling the complex electronic structure of amorphous metal oxides. Proc. Natl Acad. Sci. USA 108, 6355-6360 (2011).

5. Kim, Y.-H. et al. Flexible metal-oxide devices made by room-temperature photochemical activation of sol-gel films. Nature 489, 128-132 (2012).

6. Idota, Y., Kubota, T., Matsufuji, A., Maekawa, Y.\& Miyasaka, T. Tin-based amorphous oxide: a high-capacity lithium-ion-storage material. Science 276, 1395-1397 (1997).

7. Granqvist, C. G. Handbook of Inorganic Electrochromic Materials (Elsevier Science, 2002).

8. Tangirala, R., Baker, J. L., Alivisatos, A. P. \& Milliron, D. J. Modular inorganic nanocomposites by conversion of nanocrystal superlattices. Angew. Chem. Int. Ed. 49, 2878-2882 (2010)

9. Llordes, A. etal. Polyoxometalates and colloidal nanocrystals as building blocks for metal oxide nanocomposite films. J. Mater. Chem. 21, 11631-11638 (2011).

10. Rosenflanz, A. et al. Bulk glasses and ultrahard nanoceramics based on alumina and rare-earth oxides. Nature 430, 761-764 (2004)

11. Falcão-Filho, E. L. et al. Third-order optical nonlinearity of a transparent glass ceramic containing sodium niobate nanocrystals. Phys. Rev. B 69, 134204 (2004)

12. Mattarelli, M., Gasperi, G., Montagna, M. \& Verrocchio, P. Transparency and longranged fluctuations: the case of glass ceramics. Phys. Rev. B 82, 094204 (2010).
13. Schirmeisen, A. et al. Fast interfacial ionic conduction in nanostructured glass ceramics. Phys. Rev. Lett. 98, 225901 (2007).

14. Zhou, H. S., Li, D. L., Hibino, M. \& Honma, I. A self-ordered, crystalline-glass, mesoporous nanocomposite for use as a lithium-based storage device with both high power and high energy densities. Angew. Chem. Int. Ed. 44, 797-802 (2005).

15. Dong, W. et al. Controllable and repeatable synthesis of thermally stable anatase nanocrystal-silica composites with highly ordered hexagonal mesostructures. J. Am. Chem. Soc. 129, 13894-13904 (2007).

16. Sakamoto, A. \& Yamamoto, S. Glass-ceramics: engineering principles and applications. Int. J. Appl. Glass Sci. 1, 237-247 (2010).

17. Dong, A. et al. A generalized ligand-exchange strategy enabling sequential surface functionalization of colloidal nanocrystals. J. Am. Chem. Soc. 133, 998-1006 (2011).

18. Rosen, E. L. et al. Exceptionally mild reactive stripping of native ligands from nanocrystal surfaces by using Meerwein's salt. Angew. Chem. Int. Ed. 51, 684-689 (2012).

19. McConnell, A. A., Anderson, J. S. \& Rao, C. N. R. Raman-spectra of niobium oxides. Spectrochim. Acta 32, 1067-1076 (1976).

20. Jehng, J. M. \& Wachs, I. E. Structural chemistry and Raman-spectra of niobium oxides. Chem. Mater. 3, 100-107 (1991).

21. Shelby, J. E. Introduction to Glass Science and Technology Ch. 2, 5 (Royal Society of Chemistry, 2005)

22. Monk, P., Mortimer, R. \& Rosseinsky, D. Electrochromism and Electrochromic Devices Ch. 2 (Cambridge Univ. Press, 2007).

23. Li, S.-Y., Niklasson, G. A. \& Granqvist, C. G. Nanothermochromics: calculations for $\mathrm{VO}_{2}$ nanoparticles in dielectric hosts show much improved luminous transmittance and solar energy transmittance modulation. J. Appl. Phys. 108, 063525 (2010).

24. Garcia, G. et al. Dynamically modulating the surface plasmon resonance of doped semiconductor nanocrystals. Nano Lett. 11, 4415-4420 (2011).

25. Garcia, G. et al. Near-infrared spectrally selective plasmonic electrochromic thin films. Adv. Opt. Mater. 1, 215-220 (2013).

26. Rosario, A. V. \& Pereira, E. C. Influence of the crystallinity on the $\mathrm{Li}+$ intercalation process in $\mathrm{Nb}_{2} \mathrm{O}_{5}$ films. J. Solid State Electrochem. 9, 665-673 (2005).

27. Wang, R. Y., Tangirala, R., Raoux, S., Jordan-Sweet, J. L. \& Milliron, D. J. Ionic and electronic transport in $\mathrm{Ag}_{2} \mathrm{~S}$ nanocrystal- $\mathrm{GeS}_{2}$ matrix composites with sizecontrolled $\mathrm{Ag}_{2} \mathrm{~S}$ nanocrystals. Adv. Mater. 24, 99-103 (2012).

28. Lehn, J. M. Supramolecular chemistry-receptors, catalysis, and carriers. Science 227, 849-856 (1985)

29. Li, H., Eddaoudi, M., O'Keeffe, M. \& Yaghi, O. M. Design and synthesis of an exceptionally stable and highly porous metal-organic framework. Nature $\mathbf{4 0 2}$ 276-279 (1999)

30. Buonsanti, R. et al. Assembly of ligand-stripped nanocrystals into precisely controlled mesoporous architectures. Nano Lett. 12, 3872-3877 (2012).

Supplementary Information is available in the online version of the paper.

Acknowledgements We thank S. Raoux and J. L. Jordan-Sweet as well as S. Mannsfeld and $\mathrm{M}$. Toney for assistance in synchrotron XRD measurements at the National Synchrotron Light Source (Brookhaven National Laboratory) and Stanford Synchrotron Radiation Lightsource (SSRL); and R. Zuckermann, P. J. Schuck, R. J. Mendelsberg, and especially M. Salmeron and O. Yaghi for critical reading of the manuscript. This work was performed at the Molecular Foundry, Lawrence Berkeley National Laboratory, and was supported by the Office of Science, Office of Basic Energy Sciences, of the US Department of Energy (DOE) under contract number DE-AC0205CH11231. D.J.M. and G.G. were supported by a DOE Early Career Research Program grant under the same contract, and J.G. was supported by Consejo Superior de Investigaciones Cientificas, CSIC, JAE. Scanning transmission electron microscopy images were taken at Oak Ridge National Laboratory (ORNL), supported by DOE-BES, Materials Sciences and Engineering Division, and by ORNL's Shared Research Equipment (ShaRE) User Program, which is also sponsored by DOE-BES. XRD data shown in the manuscript was acquired at SSRL, beamline 11-3.

Author Contributions A.L. synthesized the materials, carried out the experiments and analysed the data, with assistance from G.G. for the electrochemical characterization. J.G. carried out scanning transmission electron microscopy imaging. A.L. and D.J.M. were responsible for experimental design and wrote the manuscript, which incorporates critical input from all authors.

Author Information Reprints and permissions information is available at www.nature.com/reprints. The authors declare competing financial interests: details accompany the full-text HTML version of the paper at www.nature.com/nature. Readers are welcome to comment on the online version of the paper. Correspondence and requests for materials should be addressed to D.J.M. (dmilliron@lbl.gov). 


\section{METHODS}

Nanocrystal-in-glass (ITO-in-NbO ${ }_{x}$ ) composite films were prepared from aqueous colloidal solutions containing polyoxometalate clusters bonded to the nanocrystals surface. On solution deposition (spin coating) and thermal annealing $\left(400{ }^{\circ} \mathrm{C}\right.$ in air), polyoxometalates condense into an amorphous oxide matrix, which remains covalently linked to the embedded nanocrystals. The volume fraction of nanocrystals in the nanocomposite films was tuned by varying the polyoxometalate molar concentration in the initial colloidal solution. XRD and scanning transmission electron microscopy were used for structural characterization of the films at different stages of the thermal process. In addition, films with different nanocrystal interfacial areas were analysed by Raman spectroscopy to systematically follow structural changes in the amorphous matrix induced by the presence of nanocrystals. In parallel, the electrochromic properties were studied by means of spectroelectrochemical measurements. Transmittance spectra were acquired in situ, under applied voltage, by placing the film as working electrode in a liquid spectroelectrochemical cell ( $\mathrm{Li}$ foils as counter/reference electrodes and $\mathrm{LiClO}_{4} /$ propylene carbonate as electrolyte).

Synthesis of POMs and ITO nanocrystals. We used two different POMs; the hexaniobates $\left[\mathrm{Nb}_{6} \mathrm{O}_{19}\right]^{8-}$ and decaniobates $\left[\mathrm{Nb}_{10} \mathrm{O}_{28}\right]^{6-}$ were both prepared using a solution precipitation method with tetramethylammonium, $\left[\mathrm{N}\left(\mathrm{CH}_{3}\right)_{4}\right]^{+}$, as counterion. We recently reported the synthesis and detailed characterization of these $\mathrm{POMs}^{9}$. Briefly, to synthesize decaniobate clusters, $3.19 \mathrm{~g}(10 \mathrm{mmol})$ of niobium ethoxide $\left(\mathrm{Nb}(\mathrm{OEt})_{5}, 99.99 \%\right.$, Alfa Aesar) were added to a flask containing $25 \mathrm{ml}$ ethanol Then, $1.81 \mathrm{~g}(10 \mathrm{mmol})$ tetramethylammonium hydroxide $\left(\mathrm{N}\left(\mathrm{CH}_{3}\right)_{4} \mathrm{OH} \cdot 5 \mathrm{H}_{2} \mathrm{O}\right.$, $97 \%$, Aldrich) were added to the flask and the reaction mixture was refluxed overnight, then cooled to room temperature and centrifuged. The resulting solid product was washed with $\mathrm{H}_{2} \mathrm{O}$ and precipitated with acetone (three times). After drying overnight under vacuum at room temperature, a crystalline white powder was collected ( $47 \%$ reaction yield). XRD of the resultant powder as well as thermogravimetry coupled to mass spectrometry confirmed the expected chemical formula: $\left[\mathrm{N}\left(\mathrm{CH}_{3}\right)_{4}\right]_{6} \mathrm{Nb}_{10} \mathrm{O}_{28} \cdot 6 \mathrm{H}_{2} \mathrm{O}$ (ref. 9). The hexaniobates, $\left[\mathrm{Nb}_{6} \mathrm{O}_{19}\right]^{8-}$, were synthesized by dissociating decaniobate clusters at $\mathrm{pH} 14$. The reaction was performed at room temperature by mixing $0.21 \mathrm{mmol}\left[\mathrm{N}\left(\mathrm{CH}_{3}\right)_{4}\right]_{6} \mathrm{Nb}_{10} \mathrm{O}_{28} \cdot 6 \mathrm{H}_{2} \mathrm{O}$ and $1.48 \mathrm{mmol}$ $\mathrm{N}\left(\mathrm{CH}_{3}\right)_{4} \mathrm{OH} \cdot 5 \mathrm{H}_{2} \mathrm{O}$ in a flask containing $10 \mathrm{ml} \mathrm{EtOH} / \mathrm{H}_{2} \mathrm{O}(1: 1)$. After $2 \mathrm{~h}$ stirring, the solid product, with the expected chemical formula $-\left[\mathrm{N}\left(\mathrm{CH}_{3}\right)_{4}\right]_{8} \mathrm{Nb}_{6} \mathrm{O}_{19} \cdot 15$ $\mathrm{H}_{2} \mathrm{O}$-was precipitated with acetone and dried overnight under vacuum. Given that the $\mathrm{pH}$ stability of hexaniobates is within the range of $12.5-14$, we avoided further washing, which would induce its condensation to decaniobate clusters $(\text { stable at neutral } \mathrm{pH})^{31}$.

ITO nanocrystals, with sizes ranging from $5 \mathrm{~nm}$ to $15 \mathrm{~nm}$ and atomic percentage of $\mathrm{Sn}$ doping from 4 at.\% to 12 at.\%, were synthesized by following established colloidal methods ${ }^{24,32}$. The size of the nanocrystals was determined by collecting statistics in bright-field transmission electron microscope images, and atomic doping concentration was assessed by inductively coupled plasma atomic emission spectroscopy. The ITO-in- $\mathrm{NbO}_{x}$ composite films that we used for electrochromic properties testing and Raman characterization contained ITO nanocrystals with Sn at.\% of $12.2 \pm 0.1$ and diameter $4.8 \mathrm{~nm} \pm 0.1 \mathrm{~nm}$.

Linking process for POM-stabilized nanocrystals. As-synthesized ITO nanocrystals were stripped of their coordinated organic ligands and their naked, metaladatom-rich surfaces ${ }^{17,18}$ were further functionalized with POMs. The chemical link between POMs and the nanocrystals is made by bonding nucleophilic POM oxygens to In/Sn adatoms in ITO.

In the ligand-stripping process, a two-phase mixture was prepared in a vial by combining equal volumes of $N, N$-dimethylformamide (DMF) and organic-capped ITO $\left(\sim 60 \mathrm{mg} \mathrm{ml}^{-1}\right)$ dispersed in hexane. Then, $\sim 7 \mathrm{mg}$ nitrosonium tetrafluoroborate $\left(\mathrm{NOBF}_{4}, 97 \%\right.$, Acros) were added and the resulting mixture was either vigorously stirred or sonicated. After 5-10 min, ITO nanocrystals were quantitatively transferred to the polar DMF phase as a result of their organic-free surface, now weakly coordinated to the solvent (DMF) molecules. These naked nanocrystals were precipitated with toluene and the supernatant removed by centrifugation. After further purification with toluene and DMF (three washings), the nanocrystals were dispersed in DMF (final concentration, $60 \mathrm{mg} \mathrm{ml}^{-1}$ ). Next, $1 \mathrm{ml}$ of an aqueous solution of hexaniobate clusters, $\left[\mathrm{N}\left(\mathrm{CH}_{3}\right)_{4}\right]_{8} \mathrm{Nb}_{6} \mathrm{O}_{19}$ (concentration varies depending on the targeted final volume fraction, typically $200 \mathrm{mg} \mathrm{m}^{-1}$ ) were added to $1 \mathrm{ml}$ DMF ITO dispersion, which instantly turned opaque. After vigorously stirring ( $30 \mathrm{~min}$ ), the slurry becomes clear as a result of POMs bonding to the naked ITO nanocrystals, which now become perfectly soluble in water (Fig. 1a). The colloidal solution was washed three times with $\mathrm{DMF} / \mathrm{H}_{2} \mathrm{O}$ to ensure complete removal of DMF molecules. The resultant POM-ITO pellet was redispersed in ultrapure water. Note that hexaniobates were found to be more effective than decaniobates in coordinating the bare nanocrystal surfaces to form stable aqueous dispersions. We ascribe this behaviour to the higher charge density of hexaniobates, which facilitates the surface coverage and extent of linking to the nanocrystal.

Addition of excess POM (that is, decaniobates) to the POM-ITO solution allowed us to vary the volume fraction of $\mathrm{NbO}_{x}$ glass in the final ITO-in- $\mathrm{NbO}_{x}$ film Aqueous POM-ITO solutions are perfectly stable and no ageing has been observed after two years following preparation. Ethanol was added as co-solvent to increase the viscosity of the solution and thus improve the quality of the deposited film.

Preparation of ITO-in- $\mathrm{NbO}_{x}$ nanocrystal-in-glass films. POM-ITO colloidal solutions, in water/ethanol (1:1), were deposited by spin coating onto a substrate (such as Si or ITO-coated glass) which had been previously cleaned and treated with ultraviolet-ozone to ensure a hydrophilic surface. As-deposited POM-ITO films were then annealed at $400{ }^{\circ} \mathrm{C}$ for $20 \mathrm{~min}$, in air, using a rapid thermal annealing furnace at $10{ }^{\circ} \mathrm{C} \mathrm{s}^{-1}$.

XRD of POM-ITO and ITO-in-NbO ${ }_{x}$. The atomic structure of as-deposited POM-ITO (Supplementary Fig. 3) and annealed ITO-in-NbO ${ }_{x}$ films (Fig. 2b-d, Supplementary Fig. 5) was assessed by XRD with synchrotron radiation $(\lambda=0.974 \AA$ ) at the Stanford Synchrotron Radiation Lightsource (SSRL), beamline 11-3. Acquisitions were performed in grazing incident mode (incidence angle of $0.12^{\circ}$ ) and the scattered X-rays collected with an area detector.

Transmission electron microscopy of POM-ITO and ITO-in-NbO ${ }_{x}$. Transmission electron microscope images of the POM-ITO colloidal solutions (Supplementary Fig. 2) were acquired on a JEOL-2100 with a $\mathrm{LaB}_{6}$ filament at $200 \mathrm{kV}$ and equipped with a Gatan camera. Samples for transmission electron microscope analysis were prepared by drying a drop of POM-ITO aqueous solution on the surface of a carbon-coated copper grid, which had been previously treated with ultraviolet-ozone.

Cross-sectional scanning transmission electron microscopy images of ITO-in$\mathrm{NbO}_{x}$ films (Fig. 1e, f) were carried out in a Nion UltraStem operated at $100 \mathrm{kV}$ and equipped with a Nion aberration corrector. A high-angle annular dark field (HAADF) detector allowed us to record incoherent $Z$-contrast images, in which the intensity of atom columns directly reflects their mean square atomic number (Z). Specimens for scanning transmission electron microscopy were prepared by conventional methods, that is, grinding, dimpling and Ar ion milling.

Scanning electron microscopy and energy dispersive spectroscopy. Scanning electron microscope imaging (Supplementary Fig. 6) was carried out on a Zeiss Gemini Ultra-55 microscope, using beam energies of $5-10 \mathrm{kV}$ and an In-Lens detector. Film thickness was determined by cross-sectional imaging. An inbuilt energy dispersive spectroscopy detector was used for elemental analysis. The volume fraction of each component in the final nanocrystal-in-glass films was estimated from the measured metal atomic ratios and their bulk densities.

Raman spectroscopy of ITO-in- $\mathbf{N b O}_{x}$ films. The bonding arrangement of the amorphous $\mathrm{NbO}_{x}$ matrix was studied by Raman spectroscopy (Fig. 2), using a Horiba LabRAM Aramis instrument with confocal aperture. All Raman spectra were obtained with a $\times 100$ microscope objective (numerical aperture of 0.90 ), $17.7 \mathrm{~mW}$ of laser power at an excitation wavelength of $532 \mathrm{~nm}$, and an acquisition time of $300 \mathrm{~s}$. The spectral resolution was $1.5 \mathrm{~cm}^{-1}$ and the laser spot size was $\sim 721 \mathrm{~nm}$. Several spots were examined on each sample and showed similar spectra. Raman peaks from the substrate (ITO-sputtered glass slides) appeared in a different spectral region than $\mathrm{NbO}_{x}$, allowing accurate data analysis and interpretation. Peak-fitting analysis was carried out in OriginPro software.

Optical switching and electrochemical measurements of ITO-in- $\mathrm{NbO}_{x}$ films. Optical switching properties at different voltages (Fig. 4) were studied in a homebuilt spectroelectrochemical cell, in which the ITO-in- $\mathrm{NbO}_{x}$ film was placed as the working electrode and in situ transmission visible-NIR spectra were recorded as functions of the applied potential $\left(1.5 \mathrm{~V}-4 \mathrm{~V}\right.$ versus $\left.\mathrm{Li} / \mathrm{Li}^{+}\right)$. The spectroelectrochemical cell had a three-electrode configuration, with Li foils as counter and reference electrodes, the electrolyte being $0.1 \mathrm{M} \mathrm{LiClO}_{4}$ in anhydrous propylene carbonate.

The electrochemical stability was evaluated by cyclic voltammetry, in which the voltages were swept between the potential limits at $30 \mathrm{mV} \mathrm{s}^{-1} 2,000$ times. Spectroelectrochemical and cyclic voltammetry measurements were performed in an argon glove box with a ASD Quality Spec Pro visible-NIR spectrometer and a Bio-logic VSP potentiostat. ITO-in- $\mathrm{NbO}_{x}$ films were deposited on conducting glass substrates (that is, ITO-sputtered glass slides) to ensure good in-plane conductivity.

31. Villa, E. M. et al. Reaction dynamics of the decaniobate ion $\left[\mathrm{H}_{\mathrm{x}} \mathrm{Nb}_{10} \mathrm{O}_{28}\right]^{(6-\mathrm{x})-}$ in water. Angew. Chem. Int. Edn 47, 4844-4846 (2008).

32. Choi, S. I., Nam, K. M., Park, B. K., Seo, W. S. \& Park, J. T. Preparation and optical properties of colloidal, monodisperse, and highly crystalline ITO nanoparticles. Chem. Mater. 20, 2609-2611 (2008). 